\title{
GxE Interaction Analysis of Wheat Genotypes Evaluated under Restricted Irrigated Timely Sown Conditions of North Eastern Plains Zone using AMMI \& Yield Stability Measures
}

\author{
Ajay Verma* and G.P. Singh \\ ICAR-Indian Institute of Wheat \& Barley Research, Karnal, Haryana, India \\ *Corresponding author
}

\section{A B S T R A C T}

Keywords

AMMI analysis, ASV, SIPC, Za, EV, SI, SSI, Biplot graphs

Article Info

Accepted:

10 October 2020 Available Online: 10 November 2020
Highly significant effects of environment (E), GxE interaction and genotypes (G) observed by AMMI analysis during 2018-19 and 2019-20 study years. Stable genotypes deviates minimum from the average performance across environments would be assessed by lower values of WAASB measure. WAASB measure observed suitability of HI1612 \& K1317 wheat genotypes during 2018-19. Superiority index found K8027 \& HD2888 as of stable performance with high yield. More over the average yield of genotypes ranked HD3293 \& HI1612 as of order of choice. PRVG and MHPRVG measures settled for HI1612 \& HD3293 wheat genotypes. Only indirect relations were observed with stability measures (EV, SIPC, by SI measure otherwise positive values of correlation were seen. WAASB measure exhibited indirect relationships with other EV, yield, MHPRVG \& PRVG along with positive values for remaining stability measures. Second year of study observed suitability of HI1612 \& K1317 wheat genotypes by WAASB measure. Superiority index while weighting 0.65 and 0.35 for yield \& stability found HI1612 \& K1317 as of stable performance along with high yield. PRVG as well as MHPRVG measures observed suitability of HI1612, \& K1317 while DBW252 as unstable wheat genotypes. More over the average yield of genotypes ranked HI1612 \& K1317 as of order of choice. Values of SI measure had expressed only indirect relations of high degree with measures except with EV, SIPC, yield, PRVG and MHPRVG values. WAASB had positive relations with AMMI based measures and of negative correlation values with SI, yield, PRVG and MHPRVG values.

\section{Introduction}

Wide use of AMMI model, hybrid of additive and multiplicative components, to separates the additive variance from the multiplicative variance and application of principal component analysis (PCA) to the interaction portion (Gauch, 2013; Bocianowski et al.,
2019). This analysis has been proved to be an effective process to captures a large portion of the GxE interaction sum of squares, thereby separating main and interaction effects (Ajay et al., 2019).

Multi Environment trials of all crops demand an efficient estimation of main and interaction 
effects (Bornhofen et al., 2017). More over biased interpretation regarding the stability of the genotypes had been also reported when low proportion of the variance explained by first interaction principal component IPCA1 under AMMI analysis (Ramburan et al., 2011; Zali et al., 2012; Oyekunle et al., 2017).

The quantitative stability measure i.e. Weighted Average of Absolute scores (WAASB), as an important statistical tool recommended for identifying productive genotypes with broad adaptation (Olivoto, 2018).

The most stable genotype possessed the lower value of WAASB measure i.e. deviates minimum from the mean performance across environments (Olivoto, 2019).

The selection of promising genotypes had been assisted by use of superiority index (WAASBY) that is the simultaneous use of yield and stability by variable weighting mechanism for yield and stability of genotypes (Olivoto et al., 2019). The prime objective of the present study was to validate the type of relationships between WAASBY and other stability measures, as per AMMI model, of wheat genotypes evaluated under multi environmental trials in the North Eastern Plains Zone of the country under restricted irrigated timely sown trials in the recent past.

\section{Materials and Methods}

Bihar, eastern Uttar Pradesh, Jharkhand, Assam and plains of West Bengal comprises the North Eastern Plains Zone of India.

This zone has potential to increase the wheat production of the country as importance of this zone has been highlighted to ensure food security of the country. Eight advanced wheat genotypes sixteen locations and six genotypes at thirteen locations were evaluated under field trials at of north eastern plains zone during 2018-19 and 2019-20 cropping seasons respectively.

Field trials were conducted at research centers in randomized complete block designs with three replications. Recommended agronomic practices were followed to harvest good yield.

Details of genotype parentage along with environmental conditions were reflected in tables 1 \& 2 for ready reference. Stability measure Weighted Average of Absolute Scores has been calculated as

$\mathrm{WAASB}=\sum_{k=1}^{p}\left|I P C A_{i k} \times E P_{k}\right| / \sum_{k=1}^{p} \quad E P_{k}$

where $\mathrm{WAASB}_{\mathrm{i}}$ is the weighted average of absolute scores of the $i$ th genotype (or environment); IPCA $i k$ is the score of the $i$ th genotype (or environment) in the $k$ th IPCA, and $\mathrm{EP}_{k}$ is the amount of the variance explained by the $k$ th IPCA.

Superiority index allows weighting between yield and stability measure (WAASB) to select genotypes that combine high performance and stability as SI= $\underline{\left(r G_{i} \times \theta_{Y}\right\}+\left(r W W_{i} \times \theta_{S}\right)}$

$\left(\theta_{Y}+\theta_{S}\right) ;$ where $r G_{i}$ and $r W_{i}$ are the rescaled values for yield and WAASB, respectively, for the $i$ th genotype; $G_{i}$ and $W_{i}$ are the yield and the WAASB values for $i$ th genotype.

SI superiority index for the $i$ th genotype that weights between yield and stability, and $\theta Y$ and $\theta S$ are the weights for yield and stability assumed to be of order 65 and 35 respectively in this study. 


\begin{tabular}{|c|c|c|}
\hline Zobel & $\begin{array}{l}\text { Averages of the squared } \\
\text { eigenvector values }\end{array}$ & $E V=\sum_{n=1}^{N} \lambda_{i n}^{2} / n$ \\
\hline Sneller et al & $\begin{array}{l}\text { Sums of the absolute value } \\
\text { of the IPC scores }\end{array}$ & $S I P C=\sum_{n=1}^{\infty} \lambda_{n}^{0.5} \gamma_{i n}$ \\
\hline Purchase & AMMI stability Value & $\left.\mathrm{ASV}=\left[\frac{\operatorname{SSIPC1}}{\operatorname{SSIPC2}} \mathrm{PCl}\right)^{2}+(P C 2)^{2}\right]^{1 / 2}$ \\
\hline Rao and Prabhakaran & $\begin{array}{l}\text { AMMI based stability } \\
\text { parameter }\end{array}$ & $A S T A B=\sum_{n=1}^{n} \quad \lambda_{n} \gamma_{n i}^{2}$ \\
\hline Zali et al & ASV1 & ASV1 $\left.=\frac{\operatorname{SSIPC1}}{\operatorname{SEFC2}}(P C I)^{2}+(P C 2)^{2}\right]^{1 / 2}$ \\
\hline Zali et al & $\begin{array}{l}\text { Modified AMMI stability } \\
\text { Value }\end{array}$ & MASV $=\sqrt{\sum_{n=1}^{N-1} \frac{\operatorname{SSIPC}_{n}}{\operatorname{SSIPC} C_{n+1}}\left(P C_{n}\right)^{2}+\left(P C_{n+1}\right)^{2}}$ \\
\hline Zali et al & $\begin{array}{l}\text { Absolute value of the } \\
\text { relative contribution of IPCs } \\
\text { to the interaction }\end{array}$ & $Z_{\mathrm{a}}=\sum_{\mathrm{n}=1}^{M} \quad\left\|\lambda_{\mathrm{n}} \gamma_{\mathrm{in}}\right\|$ \\
\hline Ajay et al & MASV1 & 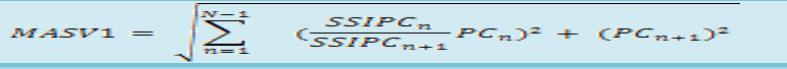 \\
\hline Resende \& Durate & $\begin{array}{l}\text { Relative performance of } \\
\text { genotypic values across } \\
\text { environments }\end{array}$ & $\mathrm{PRVG}_{\mathrm{ij}}=\mathrm{VG}_{\mathrm{ij}} / \mathrm{VG}_{\mathrm{i}}$ \\
\hline Resende \& Durate & $\begin{array}{l}\text { Harmonic mean of Relative } \\
\text { performance of genotypic } \\
\text { values }\end{array}$ & MHPRVG $_{\text {i. }}=$ Number of environments $/ \sum_{j=1}^{k_{j}} \frac{1}{p R V G_{i j}}$ \\
\hline Olivato & Superiority Index & 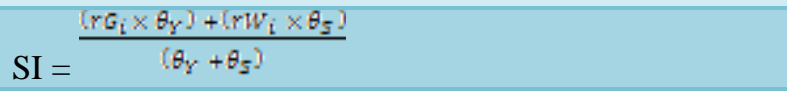 \\
\hline
\end{tabular}

AMMI analysis was performed using AMMISOFT version 1.0, available at https://scs.cals.cornell.edu/people/ hughgauch/ and SAS software version 9.3. Stability measures had been compared with recent analytic measures of adaptability calculated as the relative performance of genetic values (PRVG) and harmonic mean based measure of the relative performance of the genotypic values (MHPRVG) for the simultaneous analysis of stability, adaptability and yield (Resende and Durate, 2007).

\section{Results and Discussion}

\section{First year of study (2018-19)}

\section{AMMI analysis of MET}

The AMMI model is comprised of additive main effects of genotype and environment, and the multiplicative effect of $\mathrm{GxE}$ interaction, and thus can explain more information compared to other methods (Gauch 2013). AMMI analysis as such does not make provision for a quantitative stability measure that is deemed useful to quantify the ranking of studied genotypes according to their yield stability. AMMI stability parameters permit to evaluate yield stability after reduction of the noise from the GxE interaction effects (Zhang et al., 1998). Highly significant effects of environment (E), genotypes $(\mathrm{G})$ and $\mathrm{GxE}$ interaction had been observed by AMMI analysis.

Environment explained about significantly $56.8 \%$ of the total sum of squares due to treatments indicating that diverse environments caused most of the variations in genotypes yield (Table 3). Significant proportion of GxE interaction deserves the stability estimation of genotypes over environments (Veenstra et al., 2019). 
Genotypes explained only $112.9 \%$ of total sum of squares, whereas GxE interaction accounted for $22.4 \%$ of treatment variations in yield. More of $\mathrm{GxE}$ interaction sum of squares as compared to genotypes indicated the presence of genotypic differences across environments and complex GxE interaction for wheat yield. Further partitioning of $\mathrm{GxE}$ interaction through the AMMI model revealed that the first six multiplicative terms (IPCA1, IPCA2, IPCA3, IPCA4, IPCA5 and IPCA6) of AMMI were significant and explained $55.3 \%, 20.2 \%, 9.3 \%, 6.9 \%, 4.5 \%$ and $3.0 \%$ of interaction sum of squares, respectively. Total of significant components were $99 \%$ and remaining $1.0 \%$ is the residual or noise, which is not interpretable and thus discarded (Adjebeng et al., 2017).

\section{Stability measures behaviour}

Least value of absolute IPCA1 expressed by G2, G1 and higher value achieved by G5 (Table 5). Low values of (EV) associated with stable genotype accordingly, the genotype G6 followed by G7 and genotype G1 had the maximum value of EV measure. The lower value SIPC measure identified G2 followed by $\mathrm{G} 4$ as the most stable genotypes, whereas G8 would be of least stable behaviour. Za measure considered absolute value of the relative contribution of IPCs to the interaction revealed G2 and G3 genotypes as most stable in descending order of stability, whereas G5 genotype with the least stability. ASTAB measure observed genotypes G2 and G6 as most stable and genotype G5 was least stable in this study (Rao and Prabhakaran, 2005). ASV measure showed that genotypes G2, G3 possessed lower values would express stable performance and G5 be of least stable type. Values of ASV1 selected G2 G1 for their stable behaviour whereas G5 would express unstable performance. Measures MASV and MASV1 consider all significant IPCAs. Values of MASV showed that the genotypes,
G2 and G6 were most stable and G2 \& G4 would be stable by MASV1measure respectively (Ajay et al., 2019).

The lower values of WAASB associated with stable nature of genotypes as G2, G1 for considered locations of the zone at the same time maximum value obtained by G5, that is, the one that deviates maximum from the average performance across environments. Lower value of Superiority index had observed for G4 and G3 whereas large value by G5. Genotypes G7 and G5 were identified for their more stable yield performance by MHPRVG and PRVG measure along with least stable yield of G2. Maximum yield expressed by G3 followed by G2 and G1 as little variation had been observed from 29.1 to $38.7 \mathrm{q} / \mathrm{ha}$ among genotypes.

\section{Ranking of genotypes by simultaneous consideration of AMMI and yield}

Stability alone is not a desirable selection criterion as stable genotypes may not be a high yielders, simultaneous use of yield and stability in a single measure is essential (Kang 1993; Farshadfar et al., 2008). Simultaneous Selection Index also referred to as genotype stability index (GSI) or yield stability index (YSI) (Farshadfar et al., 2011) was computed by adding the ranks of stability measure and average yield of genotypes. As per the least values of simultaneous ranks for IPCA1 measure HI1612 \& HD3293 were considered as stable with high yield, whereas high values suggested as least stable yield for HD2888 genotype (Table 7). EV measure identified DBW252 \& HI1612 by whereas SPIC favoured HI1612 \& HD3293 genotypes. Genotypes HI1612 \& HD 3293 possessed lower value of $\mathrm{Za}$ measure. WAASB measure observed suitability of HI 1612 \& K 1317 genotypes. Superiority index while weighting 0.65 and 0.35 for yield \& stability found K 8027 \& HD2888 as of stable performance with high yield. 
Table.1 Parentage details of genotypes and environmental conditions (2018-19)

\begin{tabular}{|c|c|c|c|c|c|c|}
\hline Code & Genotype & Parentage & Environments & Latitude & Longitude & Altitude \\
\hline G 1 & K 1317 & (K0307/K9162) & Varanasi & $25^{\circ} 19^{\prime} \mathrm{N}$ & $82^{\circ} 59^{\prime} \mathrm{E}$ & 81 \\
\hline G 2 & HI 1612 & (KAUZ//ALTAR84/AOS/3/MILAN/KAUZ/4/HUITES) & Faizabad & $26^{\circ} 46^{\prime} \mathrm{N}$ & $82^{\circ} 9^{\prime} \mathrm{E}$ & 97 \\
\hline G 3 & HD 3293 & (HD2967/DBW46) & Kanpur & $26^{\circ} 26^{\prime} \mathrm{N}$ & $80^{\circ} 19^{\prime} \mathrm{E}$ & 126 \\
\hline G 4 & HD 3171 & (PBW343/HD2879) & Deegh & $26^{\circ} 02^{\prime} \mathrm{N}$ & $80^{\circ} 54^{\prime} \mathrm{E}$ & 121 \\
\hline G 5 & HD 2888 & (C306/T.SPHAEROCOCCUM//HW2004) & Ghaghraghat & $26^{\circ} 54^{\prime} \mathrm{N}$ & $81^{\circ} 56^{\prime} \mathrm{E}$ & 100 \\
\hline G 6 & DBW 252 & (PFAU/MILAN/5/CHEN/AE.SQ(TAUS)//BCN/3/VEE\#7/BOW/4/PASTOR) & IARI-Pusa & $28^{\circ} 38^{\prime} \mathrm{N}$ & $77^{\circ} 09^{\prime} \mathrm{E}$ & 52 \\
\hline G 7 & K 8027 & $(\mathrm{HD} 1696 / 2 * \mathrm{~K} 852)$ & Sabour & $25^{\circ} 23^{\prime} \mathrm{N}$ & $87^{\circ} 04^{\prime} \mathrm{E}$ & 46 \\
\hline \multirow[t]{9}{*}{ G 8} & DBW 273 & (FRANCOLIN \# $1 * 2 / /$ ND 643/2* WBLLI) & Purnea & $25^{\circ} 46^{\prime} \mathrm{N}$ & $87^{\circ} 28^{\prime} \mathrm{E}$ & 36 \\
\hline & & & RPCAU-Pusa & $25^{\circ} 98^{\prime} \mathrm{N}$ & $25^{\circ} 67 \mathrm{E}$ & 52 \\
\hline & & & Kalyani & $22^{\circ} 58^{\prime} \mathrm{N}$ & $88^{\circ} 26^{\prime} \mathrm{E}$ & 11 \\
\hline & & & Burdwan & $23^{\circ} 13^{\prime} \mathrm{N}$ & $87^{\circ} 51^{\prime} \mathrm{E}$ & 30 \\
\hline & & & Coochbehar & $26^{\circ} 8^{\prime} \mathrm{N}$ & $91^{\circ} 43^{\prime} \mathrm{E}$ & 86 \\
\hline & & & Ranchi & $23^{\circ} 20^{\prime} \mathrm{N}$ & $85^{\circ} 18^{\prime} \mathrm{E}$ & 651 \\
\hline & & & Chianki & $23^{\circ} 45^{\prime} \mathrm{N}$ & $85^{\circ} 30^{\prime} \mathrm{E}$ & 215 \\
\hline & & & KVK-Gumla & & & \\
\hline & & & Shillongani & $26^{\circ} 8^{\prime} \mathrm{N}$ & $91^{\circ} 43^{\prime} \mathrm{E}$ & 86 \\
\hline
\end{tabular}

Table.2 Parentage details of genotypes and environmental conditions (2019-20)

\begin{tabular}{|c|c|c|c|c|c|c|}
\hline Code & Genotype & Parentage & Environments & Latitude & Longitude & Altitude \\
\hline G 1 & HD3171 & (PBW343/HD2879) & Varanasi & $25^{\circ} 19^{\prime} \mathrm{N}$ & $82^{\circ} 59^{\prime} \mathrm{E}$ & 81 \\
\hline G 2 & HD2888 & (C306/T.SPHAEROCOCCUM//HW2004) & Kanpur & $26^{\circ} 26^{\prime} \mathrm{N}$ & $80^{\circ} 19^{\prime} \mathrm{E}$ & 126 \\
\hline G 3 & HD3293 & (HD2967/DBW46) & Ghaghraghat & $26^{\circ} 54^{\prime} \mathrm{N}$ & $81^{\circ} 56^{\prime} \mathrm{E}$ & 100 \\
\hline G 4 & K1317 & $(\mathrm{K} 0307 / \mathrm{K} 9162)$ & IARI-Pusa & $28^{\circ} 38^{\prime} \mathrm{N}$ & $77^{\circ} 09^{\prime} \mathrm{E}$ & 52 \\
\hline G 5 & HI1612 & (KAUZ//ALTAR84/AOS/3/MILAN/KAUZ/4/HUITES) & Sabour & $25^{\circ} 23^{\prime} \mathrm{N}$ & $87^{\circ} 04^{\prime} \mathrm{E}$ & 46 \\
\hline \multirow[t]{10}{*}{ G 6} & DBW252 & (PFAU/MILAN/5/CHEN/AE.SQ(TAUS)//BCN/3/VEE\#7/BOW/4/PASTOR) & Purnea & $25^{\circ} 46^{\prime} \mathrm{N}$ & $87^{\circ} 28^{\prime} \mathrm{E}$ & 36 \\
\hline & & & RPCAU-Pusa & $25^{\circ} 98^{\prime} \mathrm{N}$ & $25^{\circ} 67 \mathrm{E}$ & 52 \\
\hline & & & Dumka & $24^{\circ} 16^{\prime} \mathrm{N}$ & $87^{\circ} 14^{\prime} \mathrm{E}$ & 137 \\
\hline & & & Kalyani & $22^{\circ} 58^{\prime} \mathrm{N}$ & $88^{\circ} 26^{\prime} \mathrm{E}$ & 11 \\
\hline & & & Burdwan & $23^{\circ} 13^{\prime} \mathrm{N}$ & $87^{\circ} 51^{\prime} \mathrm{E}$ & 30 \\
\hline & & & Coochbehar & $26^{\circ} 34^{\prime} \mathrm{N}$ & $89^{\circ} 44^{\prime} \mathrm{E}$ & 42 \\
\hline & & & Ranchi & $23^{\circ} 20^{\prime} \mathrm{N}$ & $85^{\circ} 18^{\prime} \mathrm{E}$ & 644 \\
\hline & & & Chianki & $23^{\circ} 45^{\prime} \mathrm{N}$ & $85^{\circ} 30^{\prime} \mathrm{E}$ & 215 \\
\hline & & & Gumla & & & \\
\hline & & & Shillongani & $26^{\circ} 8^{\prime} \mathrm{N}$ & $91^{\circ} 43^{\prime} \mathrm{E}$ & 86 \\
\hline
\end{tabular}


Table.3 AMMI analysis of wheat genotypes (2018-19)

\begin{tabular}{|c|c|c|c|c|c|c|}
\hline Source & $\begin{array}{l}\text { Degree of } \\
\text { freedom }\end{array}$ & $\begin{array}{l}\text { Mean Sum of } \\
\text { Squares }\end{array}$ & $\begin{array}{l}\text { Level of } \\
\text { significance }\end{array}$ & $\begin{array}{l}\text { Proportional } \\
\text { contribution of factors }\end{array}$ & $\begin{array}{l}\text { GxE interaction } \\
\text { Sum of Squares }(\%)\end{array}$ & $\begin{array}{l}\text { Cumulative Sum of Squares } \\
(\%) \text { by IPCA's }\end{array}$ \\
\hline Treatments & 127 & 418.54 & $* * *$ & 92.07 & & \\
\hline Genotype (G) & 7 & 1060.87 & $* * *$ & 12.86 & & \\
\hline Environment (E) & 15 & 2185.53 & $* * *$ & 56.79 & & \\
\hline GxE interaction & 105 & 123.28 & $* * *$ & 22.42 & & \\
\hline IPC1 & 21 & 340.93 & $* * *$ & & 55.31 & 55.31 \\
\hline IPC2 & 19 & 137.38 & $* * *$ & & 20.16 & 75.47 \\
\hline IPC3 & 17 & 70.64 & $* * *$ & & 9.28 & 84.75 \\
\hline IPC4 & 15 & 59.24 & $* * *$ & & 6.86 & 91.61 \\
\hline IPC5 & 13 & 44.53 & $* * *$ & & 4.47 & 96.09 \\
\hline IPC6 & 11 & 34.67 & $* *$ & & 2.95 & 99.03 \\
\hline Residual & 9 & 13.94 & 0.31 & & & \\
\hline Error & 384 & 11.92 & & & & \\
\hline Total & 511 & 112.97 & & & & \\
\hline
\end{tabular}

Table.4 AMMI analysis of wheat genotypes (2019-20)

\begin{tabular}{|c|c|c|c|c|c|c|}
\hline Source & Degree of freedom & $\begin{array}{l}\text { Mean Sum of } \\
\text { Squares }\end{array}$ & $\begin{array}{c}\text { Level of } \\
\text { significance }\end{array}$ & $\begin{array}{c}\text { Proportional contribution } \\
\text { of factors }\end{array}$ & $\begin{array}{c}\text { GxE interaction } \\
\text { Sum of Squares }(\%)\end{array}$ & $\begin{array}{l}\text { Cumulative Sum of } \\
\text { Squares } \\
\text { (\%) by IPCA's }\end{array}$ \\
\hline Treatments & 89 & 380.30 & $* * *$ & 90.57 & & \\
\hline Genotype (G) & 5 & 878.82 & $* * *$ & 11.76 & & \\
\hline Environment (E) & 14 & 1431.38 & $* * *$ & 53.62 & & \\
\hline GxE interaction & 70 & 134.48 & $* * *$ & 25.19 & & \\
\hline IPC1 & 18 & 250.88 & $* * *$ & & 47.97 & 47.97 \\
\hline IPC2 & 16 & 127.94 & $* * *$ & & 21.75 & 69.72 \\
\hline IPC3 & 14 & 126.30 & $* * *$ & & 18.78 & 88.50 \\
\hline IPC4 & 12 & 55.40 & $* * *$ & & 7.06 & 95.56 \\
\hline Residual & 10 & 41.77 & $* * *$ & & & \\
\hline Error & 270 & 13.05 & & & & \\
\hline Total & 359 & 104.10 & & & & \\
\hline
\end{tabular}


Table.5 Measures of stability as per AMMI analysis for wheat genotypes evaluated under MET (2018-19)

\begin{tabular}{|c|c|c|c|c|c|c|c|c|c|c|c|c|c|c|}
\hline Genotype & IPCA1 & $\begin{array}{c}\text { MASV } \\
1\end{array}$ & MASV & ASV1 & ASV & $\mathrm{Za}$ & $\mathrm{EV}$ & SIPC & $\begin{array}{c}\text { ASTA } \\
\text { B }\end{array}$ & $\begin{array}{c}\text { WAAS } \\
\text { B }\end{array}$ & SI & $\begin{array}{c}\text { MHPRV } \\
\text { G }\end{array}$ & PRVG & Yield \\
\hline G 1 & 0.35 & 8.42 & 6.72 & 2.98 & 2.88 & 17.51 & 0.072 & 8.12 & 104.76 & 1.1636 & 24.89 & 1.057 & 1.075 & 38.48 \\
\hline G 2 & 0.01 & 4.87 & 4.34 & 0.73 & 0.73 & 9.94 & 0.070 & 6.43 & 50.29 & 0.5513 & 35.39 & 1.075 & 1.081 & 38.62 \\
\hline G 3 & 0.82 & 7.69 & 6.16 & 3.05 & 2.46 & 17.37 & 0.071 & 7.82 & 84.80 & 1.1944 & 22.95 & 1.069 & 1.079 & 38.67 \\
\hline G 4 & 1.61 & 7.11 & 5.67 & 4.49 & 2.78 & 19.02 & 0.067 & 7.70 & 88.84 & 1.4017 & 22.63 & 1.036 & 1.053 & 38.15 \\
\hline G 5 & 4.23 & 12.09 & 7.61 & 11.60 & 7.01 & 28.62 & 0.058 & 7.96 & 183.75 & 2.4189 & 62.67 & 0.792 & 0.836 & 29.52 \\
\hline G 6 & 2.04 & 7.59 & 5.36 & 5.84 & 3.77 & 21.54 & 0.044 & 7.73 & 80.59 & 1.6568 & 28.47 & 0.997 & 1.018 & 36.60 \\
\hline G 7 & 2.86 & 10.07 & 6.87 & 8.13 & 5.21 & 26.11 & 0.056 & 8.18 & 128.94 & 2.0960 & 71.05 & 0.787 & 0.816 & 29.17 \\
\hline G 8 & 2.95 & 10.17 & 6.91 & 8.37 & 5.34 & 27.78 & 0.063 & 9.25 & 135.32 & 2.1960 & 11.77 & 1.003 & 1.042 & 37.56 \\
\hline
\end{tabular}

Table.6 Measures of stability as per AMMI analysis for wheat genotypes evaluated under MET (2019-20)

\begin{tabular}{|c|c|c|c|c|c|c|c|c|c|c|c|c|c|c|}
\hline Genotype & IPCA1 & $\begin{array}{l}\text { MASV } \\
1\end{array}$ & MASV & ASV1 & ASV & $\mathrm{Za}$ & EV & SIPC & $\begin{array}{l}\text { ASTA } \\
\text { B }\end{array}$ & $\begin{array}{l}\text { WAAS } \\
\text { B }\end{array}$ & SI & $\begin{array}{l}\text { MHPRV } \\
\text { G }\end{array}$ & PRVG & Yield \\
\hline G 1 & 3.35 & 9.40 & 7.00 & 7.90 & 5.70 & 34.15 & 0.103 & 8.36 & 160.10 & 2.5271 & 27.74 & 0.9364 & 0.9659 & 34.76 \\
\hline G 2 & 2.99 & 6.95 & 4.92 & 6.75 & 4.66 & 21.83 & 0.046 & 4.76 & 87.50 & 1.6975 & 32.05 & 0.8865 & 0.9077 & 32.25 \\
\hline G 3 & 0.93 & 7.95 & 5.98 & 2.94 & 2.52 & 24.37 & 0.101 & 7.43 & 93.92 & 1.6286 & 70.26 & 1.0387 & 1.0566 & 37.56 \\
\hline G 4 & 1.47 & 10.96 & 7.44 & 3.23 & 2.18 & 23.52 & 0.104 & 6.51 & 113.07 & 1.6271 & 81.22 & 1.0615 & 1.0793 & 39.16 \\
\hline G 5 & 0.44 & 5.20 & 4.93 & 2.99 & 2.90 & 18.22 & 0.104 & 6.14 & 86.90 & 1.1787 & 100.00 & 1.1110 & 1.1224 & 40.20 \\
\hline G 6 & 3.20 & 7.21 & 4.90 & 7.09 & 4.80 & 21.44 & 0.042 & 4.48 & 87.91 & 1.6870 & 21.81 & 0.8486 & 0.8681 & 30.72 \\
\hline
\end{tabular}


Table.7 Simultaneous ranks of genotypes as per yield and AMMI based measures for wheat genotypes (2018-19)

\begin{tabular}{|c|c|c|c|c|c|c|c|c|c|c|c|c|c|c|}
\hline Genotype & $\begin{array}{l}\text { IPCA } \\
1\end{array}$ & $\begin{array}{l}\text { MASV } \\
1\end{array}$ & MASV & ASV1 & ASV & $\mathrm{Za}$ & $\mathrm{EV}$ & SIPC & $\begin{array}{l}\text { ASTA } \\
\text { B }\end{array}$ & $\begin{array}{l}\text { WAAS } \\
\text { B }\end{array}$ & SI & $\begin{array}{l}\text { MHPR } \\
\text { VG }\end{array}$ & PRVG & Yield \\
\hline K 1317 & 5 & 8 & 8 & 5 & 7 & 6 & 11 & 9 & 8 & 2 & 5 & 3 & 3 & 3 \\
\hline HI 1612 & 3 & 3 & 3 & 3 & 3 & 3 & 8 & 3 & 3 & 1 & 3 & 1 & 1 & 2 \\
\hline HD 3293 & 4 & 5 & 5 & 4 & 3 & 3 & 8 & 5 & 4 & 3 & 6 & 2 & 2 & 1 \\
\hline HD 3171 & 8 & 6 & 7 & 8 & 7 & 8 & 9 & 6 & 8 & 4 & 7 & 4 & 4 & 4 \\
\hline HD 2888 & 15 & 15 & 15 & 15 & 15 & 15 & 10 & 12 & 15 & 8 & 2 & 7 & 7 & 7 \\
\hline DBW 252 & 11 & 9 & 8 & 11 & 11 & 11 & 7 & 9 & 8 & 5 & 4 & 6 & 6 & 6 \\
\hline K 8027 & 14 & 14 & 14 & 14 & 14 & 14 & 10 & 15 & 14 & 6 & 1 & 8 & 8 & 8 \\
\hline DBW 273 & 12 & 12 & 12 & 12 & 12 & 12 & 9 & 13 & 12 & 7 & 8 & 5 & 5 & 5 \\
\hline
\end{tabular}

Table.8 Simultaneous ranks of genotypes as per yield and AMMI based measures for wheat genotypes (2019-20)

\begin{tabular}{|c|c|c|c|c|c|c|c|c|c|c|c|c|c|c|}
\hline Genotype & IPCA1 & $\begin{array}{l}\text { MASV } \\
1\end{array}$ & MASV & ASV1 & ASV & $\mathrm{Za}$ & $\mathrm{EV}$ & SIPC & $\begin{array}{l}\text { ASTA } \\
\text { B }\end{array}$ & $\begin{array}{l}\text { WAAS } \\
\text { B }\end{array}$ & SI & $\begin{array}{l}\text { MHPRV } \\
\mathrm{G}\end{array}$ & PRVG & Yield \\
\hline HD3171 & 10 & 9 & 9 & 10 & 10 & 10 & 8 & 10 & 10 & 6 & 5 & 4 & 4 & 4 \\
\hline HD2888 & 9 & 7 & 7 & 9 & 9 & 8 & 7 & 7 & 7 & 5 & 4 & 5 & 5 & 5 \\
\hline HD3293 & 5 & 7 & 7 & 4 & 5 & 8 & 6 & 8 & 7 & 3 & 3 & 3 & 3 & 3 \\
\hline K1317 & 5 & 8 & 8 & 5 & 3 & 6 & 8 & 6 & 7 & 2 & 2 & 2 & 2 & 2 \\
\hline HI1612 & 2 & 2 & 4 & 3 & 4 & 2 & 6 & 4 & 2 & 1 & 1 & 1 & 1 & 1 \\
\hline DBW252 & 11 & 9 & 7 & 11 & 11 & 8 & 7 & 7 & 9 & 4 & 6 & 6 & 6 & 6 \\
\hline
\end{tabular}


Table.9 Association analysis of SI with other stability measures (2018-19)

\begin{tabular}{|c|c|c|c|c|c|c|c|c|c|c|c|c|c|}
\hline Measure & MASV1 & MASV & ASV1 & ASV & $\mathrm{Za}$ & $\mathrm{EV}$ & SIPC & ASTAB & WAASB & SI & MHPRVG & PRVG & Yield \\
\hline IPCA1 & 0.875 & 0.704 & 0.991 & 0.961 & 0.943 & -0.598 & 0.559 & 0.875 & 0.967 & 0.498 & -0.835 & -0.794 & -0.782 \\
\hline MASV1 & & 0.953 & 0.928 & 0.968 & 0.933 & -0.333 & 0.723 & 0.980 & 0.929 & 0.459 & -0.784 & -0.738 & -0.737 \\
\hline MASV & & & 0.780 & 0.851 & 0.828 & -0.093 & 0.765 & 0.927 & 0.805 & 0.348 & -0.644 & -0.602 & -0.602 \\
\hline ASV1 & & & & 0.989 & 0.963 & -0.564 & 0.612 & 0.922 & 0.981 & 0.502 & -0.844 & -0.800 & -0.791 \\
\hline ASV & & & & & 0.973 & -0.524 & 0.676 & 0.950 & 0.982 & 0.484 & -0.831 & -0.785 & -0.778 \\
\hline $\mathrm{Za}$ & & & & & & -0.540 & 0.789 & 0.887 & 0.996 & 0.359 & -0.757 & -0.707 & -0.691 \\
\hline $\mathrm{EV}$ & & & & & & & -0.169 & -0.254 & -0.569 & -0.358 & 0.532 & 0.524 & 0.508 \\
\hline SIPC & & & & & & & & 0.634 & 0.734 & -0.158 & -0.276 & -0.214 & -0.193 \\
\hline ASTAB & & & & & & & & & 0.893 & 0.485 & -0.785 & -0.739 & -0.741 \\
\hline WAASB & & & & & & & & & & 0.410 & -0.793 & -0.746 & -0.730 \\
\hline SI & & & & & & & & & & & -0.880 & -0.912 & -0.922 \\
\hline MHPRVG & & & & & & & & & & & & 0.997 & 0.995 \\
\hline PRVG & & & & & & & & & & & & & 0.999 \\
\hline
\end{tabular}

Table.10 Association analysis of SI with other stability measures (2019-20)

\begin{tabular}{|c|c|c|c|c|c|c|c|c|c|c|c|c|c|}
\hline Measure & MASV1 & MASV & ASV1 & ASV & $\mathrm{Za}$ & EV & SIPC & ASTAB & WAASB & SI & $\begin{array}{l}\text { MHPRV } \\
\text { G }\end{array}$ & PRVG & Yield \\
\hline IPCA1 & 0.217 & -0.008 & 0.971 & 0.899 & 0.503 & -0.630 & -0.209 & 0.395 & 0.723 & -0.965 & -0.928 & -0.913 & -0.885 \\
\hline MASV1 & & 0.926 & 0.047 & -0.086 & 0.607 & 0.331 & 0.459 & 0.635 & 0.549 & -0.112 & 0.012 & 0.042 & 0.099 \\
\hline MASV & & & -0.115 & -0.180 & 0.669 & 0.649 & 0.721 & 0.756 & 0.528 & 0.128 & 0.298 & 0.334 & 0.388 \\
\hline ASV1 & & & & 0.977 & 0.506 & -0.589 & -0.165 & 0.413 & 0.718 & -0.941 & -0.894 & -0.879 & -0.858 \\
\hline ASV & & & & & 0.518 & -0.503 & -0.081 & 0.433 & 0.706 & -0.880 & -0.817 & -0.801 & -0.788 \\
\hline $\mathrm{Za}$ & & & & & & 0.318 & 0.738 & 0.941 & 0.960 & -0.461 & -0.237 & -0.193 & -0.156 \\
\hline $\mathrm{EV}$ & & & & & & & 0.848 & 0.464 & 0.050 & 0.690 & 0.842 & 0.865 & 0.879 \\
\hline SIPC & & & & & & & & 0.757 & 0.523 & 0.226 & 0.451 & 0.489 & 0.507 \\
\hline ASTAB & & & & & & & & & 0.878 & -0.278 & -0.058 & -0.015 & 0.036 \\
\hline WAASB & & & & & & & & & & -0.679 & -0.489 & -0.448 & -0.410 \\
\hline SI & & & & & & & & & & & 0.971 & 0.959 & 0.948 \\
\hline MHPRVG & & & & & & & & & & & & 0.999 & 0.994 \\
\hline PRVG & & & & & & & & & & & & & 0.997 \\
\hline
\end{tabular}




\section{Fig.1}

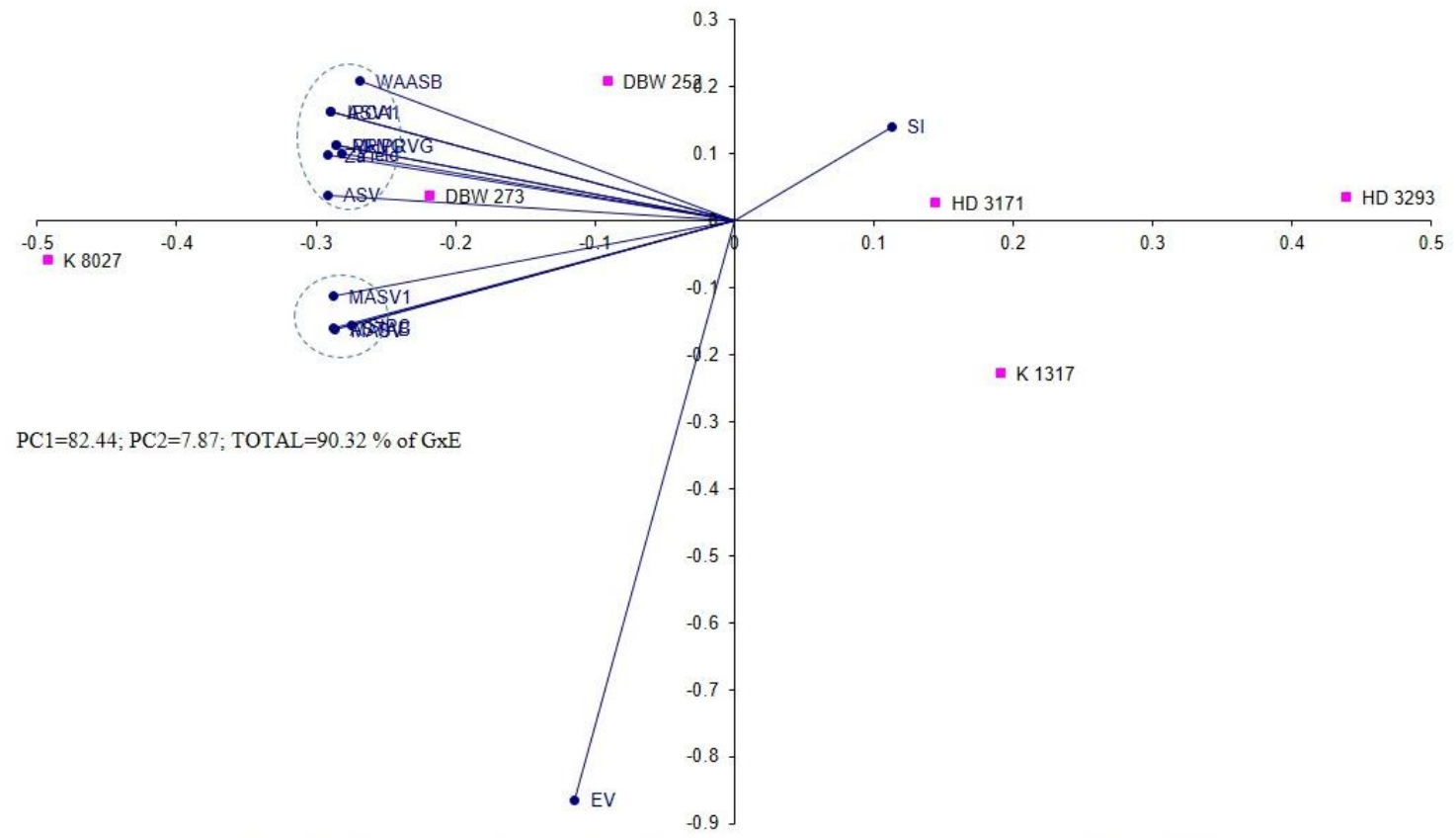

Figure 1 : Biplot graphical analysis of stability measures for wheat genotypes evaluated under MET (2018-19)

Fig.2

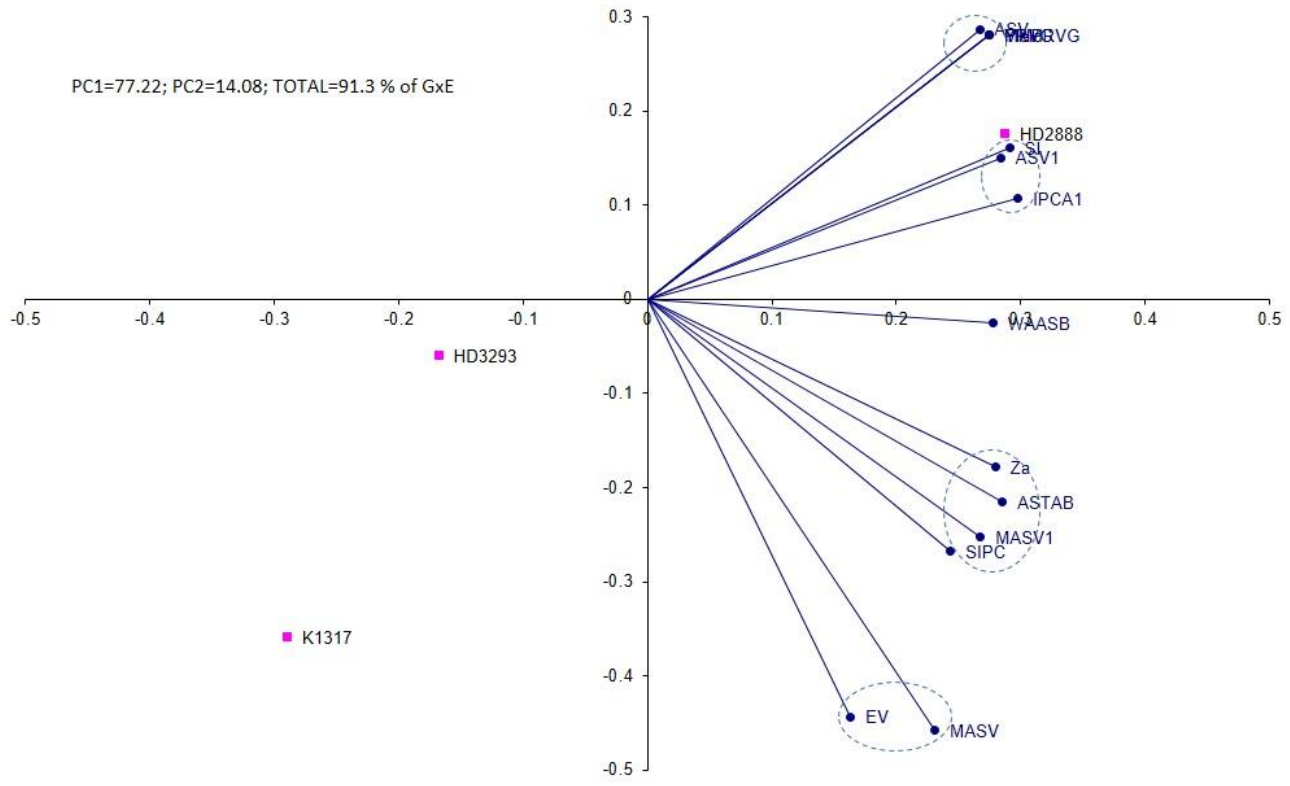

Figure 2: Biplot graphical analysis of stability measures for wheat genotypes evaluated under MET (2019-20) 
Composite measures MASV as well as MASV1 selected HI 1612, HD 3293 genotypes of choice for these locations of the zone. Values of least magnitude of ASV and ASV1 pointed towards HI 1612 and HD 3293 wheat genotypes (Oyekunle et al., 2017). In the present study, all measures identified genotypes HI 1612 and HD 3293 as stable and high yielders. PRVG and MHPRVG measures observed suitability of HI1612 \& HD3293 wheat genotypes. More over the average yield of genotypes ranked HD3293 \& HI 1612 as of order of choice.

\section{Biplot graphical pattern}

Loadings of stability measures as per first two significant principal components for evaluated wheat genotypes were reflected in table 9. Biplot graphical analysis based on two significant principal component analysis (PCA) the simultaneous ranks (Figure 1). These two PCAs accounted for $90.3 \%$ of variation of the original variables (Balestre et al., 2019). The stability measures of wheat genotypes grouped into two major groups. MASV1 clubbed with ASTAB and MASV measures. Larger group comprises of yield with ASV, IPCA1, ASV1, SIPC, Za, PRVG \& MHPRVG measures. Measure EV, and SI maintained distance from stability measures and observed in outliers in different quadrants of biplot graphical analysis.

\section{Association analysis}

Correlation values were computed for each pair of measures to have an idea about association analysis among measures. Mean yield showed only significant positive correlations with EV (Table 11). While SI and other measures expressed only negative values (IPCA1, ASV1, MASV1, ASV, WAASB) with yield. Only indirect relations were observed with stability measures (EV, SIPC) by SI measure otherwise positive values of correlation were seen. WAASB measure exhibited indirect relationships with other EV, yield, MHPRVG \& PRVG along with positive values for remaining stability measures. AMMI based measures Za, SIPC, SV, ASV1, MASV1, MASV and ASTAB achieved only positive correlation values among themselves and with others (Ajay et al 2019). More over EV had maintained negative relationships with AMMI based measures along with moderate positive values with yield, MHPRVG \& PRVG. ASTAB had indirect relation with PRVG, MHPRVG and yield. Negative correlations of SIPC with EV, SI, PRVG, MHPRVG and yield were of low magnitude. Indirect relations of $\mathrm{Za}$ observed with PRVG, MHPRVG and yield of large negative values. Same behaviour of negative correlations had displayed by IPCA1, ASV1, MASV1, ASV, MASV also.

\section{Second year of study (2019-20)}

\section{AMMI analysis of MET}

Highly significant effects of environment (E), genotypes $(\mathrm{G})$, and $\mathrm{GxE}$ interaction were seen by the AMMI analysis. Diversity of environmental effects judged by $53.6 \%$ of the total sum of squares due to treatments explained by environments only (Table 4). Genotypes explained $11.8 \%$ of total sum of squares, whereas GxE interaction explained $25.2 \%$ of treatment variation in yield. The larger magnitude of GxE interaction (sum of squares) than genotypes indicated the presence of genotypic differences across environments and complex GxE interaction for wheat yield. Further partitioning of $\mathrm{GxE}$ interaction through the AMMI analysis revealed that the first four multiplicative terms (IPCA1, IPCA2, IPCA3 and IPCA4) of AMMI were significant and explained $47.9 \%$, $21.7 \% 18.8 \%$, and $7.1 \%$ of interaction sum of squares, respectively. Approximately $95.6 \%$ of sum of squares accounted by four PC's and left over residual was discarded. 


\section{Stability measures behaviour}

Least value of absolute IPCA1 expressed by G5, G3 and higher value by G5 (Table 6). Stable performance of G6, G2 genotypes anticipated as lower values of EV were exhibited by genotypes. The sums of the absolute value of the IPC scores (SIPC) identified G6 followed by G2 as the stable genotypes, whereas $\mathrm{G} 1$ as the least stable one. Absolute value of the relative contribution of IPCs to the interaction (Za) revealed G5, and G6 genotypes as most stable in descending order of stability.

Values of ASV measure showed that genotype G4, G3 would show the stable performance and as per ASV1 selected G3 G5 for their stable behaviour. MASV selected the genotypes G6 and G were most and MASV1 considered G5 G2 would be genotypes of stable yield. AMMI-based stability parameter (ASTAB) identified genotypes G5 \& G2 as most stable as well G1 was least stable genotype in this study

Lower WAASB value of genotypes expressed least deviates from the average performance across environments.G4 \& G5 pointed by measure WAASB as desirable genotypes for considered locations of the zone. Superiority index had observed that genotypes G6 and G1 whereas maximum value achieved by G5. Stable performance of genotypes G6 G2 assured by values of PRVG measure while MHPRVG measure selected G6 G2 along with least stable yield of G5. Maximum yield expressed by G5 followed by G4 as little variation observed from 30.7 to $40.2 \mathrm{q} / \mathrm{ha}$ among genotypes.

\section{Ranking of genotypes by simultaneous consideration of AMMI and yield}

Least values of IPCA1 measure as per simultaneous ranks of genotypes HI1612 \&
HD3293 genotypes with stable high yield, whereas high values for DBW252 suggested as least stable yield (Table 8). EV measure identified HD3293 \& HI1612 for stable performance whereas as per SPIC values genotypes HI1612 \& K1317 favoured. HI1612, \& K1317 genotypes possessed lower value of $\mathrm{Za}$ measure. Least values of ASV pointed for K1317, HI1612 and ASV1 favoured HI1612 and HD3293 wheat genotypes. Modified AMMI stability Value measure MASV along with MASV1 selected HI1612, HD2888 as genotypes of choice for these locations of the zone.

WAASB measure observed suitability of HI1612 \& K1317 wheat genotypes for the locations of this zone. Superiority index while weighting 0.65 and 0.35 for yield \& stability found HI1612 \& K1317 as of stable performance along with high yield. PRVG as well as MHPRVG measures observed suitability of HI1612, \& K1317 while DBW252 as unstable wheat genotypes. More over the average yield of genotypes ranked HI1612 \& K1317 as of order of choice. More or less all measures identified HI1612, K1317 and HD3293 genotypes for stable and high yield as per considered locations of this zone.

\section{Biplot graphical pattern}

Biplot graphical analysis considered the loadings of stability measures as per first two significant principal component for evaluated wheat genotypes in order to understand the clustering pattern among measures (Table 10). The first two PCAs explained $91.3 \%$ of variation of the original variables. $\mathrm{PC} 1$ and PC2 axes distinguish measures into four groups. Small cluster of EV with MASV and another of yield with PRVG \& MHPRVG measures (Figure 2). SI clubbed with IPCA1, and ASV1. Last cluster comprises of $\mathrm{Za}$, MASV1, EV, SIPC, ASTAB measures. WAASB has been observed as an outlier. 
AMMI based measures $\mathrm{Za}$, clustered in separate cluster. Stability measures WAASB and MAVS1 were observed as outliers in biplot analysis.

\section{Association analysis}

Average yield of genotypes had positive values of higher magnitude with SI, MHPRVG and PRVG values (Table 12). However only negatives values had been observed with other measures. Similar nature of MHPRVG and PRVG measures were also seen other stability measures. Values of SI measure had expressed only indirect relations of high degree with measures except with $\mathrm{EV}$, SIPC, yield, PRVG and MHPRVG values. WAASB had positive relations with AMMI based measures and of negative correlation values with SI, yield, PRVG and MHPRVG values. AMMI based measures ASTAB, SIPC, Za, ASV, ASV1, MASV, MASV1 achieved only positive correlation values except of negative with EV. Direct correlation values had been depicted by EV along with indirect relations with IPCA1, ASV and ASV1. Values of ASTAB showed indirect relationships with SI, MHPRVG, PRVG and yield otherwise positive values were expressed.

In conclusions the AMMI model is an effective tool to study GxE interaction in multi-environment yield trials. Stability measures by simultaneous use of AMMI model and yield would be more meaning full and useful as compared to measures consider either the AMMI or yield of genotypes only. The stability measures found to be correlated well with each other. Measures MASV, MASV1, WAAB and SI could be used to identify stable high-yielding genotypes.

\section{Acknowledgements}

The wheat genotypes were evaluated at research fields at coordinated centers of
AICW\&BIP across the country. First author sincerely acknowledge the hard work of all the staff for field evaluation and data recording of wheat genotypes.

\section{References}

Adjebeng-Danquah, J., Manu-Aduening, J., Gracen V.E., Asante I.K., and Offei, S.K. 2017. AMMI stability analysis and estimation of genetic parameters for growth and yield components in cassava in the forest and guinea savannah ecologies of Ghana. Int. J. Agron. 2017:1-10.

Ajay, B. C., Aravind J., Fiyaz R. Abdul, Kumar Narendra, Lal Chuni, Gangadhar K., Kona Praveen, Dagla M. C. and Bera S. K. 2019 Rectification of modified AMMI stability value (MASV) Indian J. Genet., 79(4) 726731.

Balestre, M., Von Pinho R.G., Souza J.C., and Oliveira R.L. 2009. Genotypic stability and adaptability in tropical maize based on AMMI and GGE biplot analysis. Genet. Mol. Res. 8:1311-1322.

Bocianowski, J., Niemann J., and Nowosad K.. 2019. Genotype-by environment interaction for seed quality traits in interspecific cross-derived Brassica lines using additive main effects and multiplicative interaction model. Euphytica 215(7):1-13.

Bornhofen, E., Benin G., Storck L., Woyann L.G., Duarte T., Stoco M.G., and Marchioro S.V. 2017. Statistical methods to study adaptability and stability of wheat genotypes. Bragantia 76:1-10.

Farshadfar, E. 2008. Incorporation of AMMI stability value and grain yield in a single non-parametric index (GSI) in bread wheat. Pak. J. Biol. Sci. 11:1791-1796.

Farshadfar, E., Mahmodi N., and Yaghotipoor A. 2011. AMMI stability value and simultaneous estimation of yield and 
yield stability in bread wheat (Triticum aestivum L.). Aust. J. Crop Sci. 5:18371844.

Gauch, H.G. 2013. A simple protocol for AMMI analysis of yield trials. Crop Sci. 53:1860-1869.

Kang, M.S. 1993. Simultaneous selection for yield and stability in crop performance trials: Consequences for growers. Agronomy Journal 85:754-757.

Olivoto, T. 2018. WAASB data, Mendeley Data, v2. doi.org/10.17632/2sjz32k $3 \mathrm{~s} 3.2$

Olivoto, T. 2019. Metan: multi environment trials analysis. $\mathrm{R}$ package version 1.1.0. https://github.com/TiagoOlivoto/metan.

Olivoto, T., Lucio A. Dal'Col, Gonzalez, Silva J.A. da, and Marchioro V.S. 2019. Mean performance and stability in multi-environment trials I: Combining features of AMMI and BLUP techniques. Agron. J. 111:1-12.

Oyekunle, M., Menkir A., Mani H., Olaoye G., Usman I.S., Ado S.G. 2017. Stability analysis of maize cultivars adapted to tropical environments using AMMI analysis. Cereal Res. Commun. 45:336345.

Purchase, J.L., Hatting H. and Deventer, C.S. van. 2000. Genotype $\times$ environment interaction of winter wheat (Triticum aestivum L.) in South Africa: II. Stability analysis of yield performance. S. Afr. J. Plant Soil 17:101-107.
Ramburan, S., Zhou M. and Labuschagne M. 2011. Interpretation of genotype $\times$ environment interactions of sugarcane: Identifying significant environmental factors. Field Crops Res. 124:392-399.

Rao, A.R. and Prabhakaran V.T. 2005. Use of AMMI in simultaneous selection of genotypes for yield and stability. Journal of the Indian Society of Agricultural Statistics 59:76-82.

Resende, M.D.V. and Duarte, J.B. 2007. Precision and Quality Control in Variety Trials. Pesquisa Agropecuaria Tropical 37: 182-194.

Sneller, C.H., Norquest L. Kilgore, Dombek, D. 1997. Repeatability of yield stability statistics in soybean. Crop Sci. 37:383390.

Veenstra, L.D., Santantonio N., Jannink J.-L., and Sorrells M.E. 2019. Influence of genotype and environment on wheat grain fructan content. Crop Sci. 59:190198.

Zali, H., Farshadfar E., Sabaghpour S.H., Karimizadeh, R. 2012. Evaluation of genotype $\times$ environment interaction in chickpea using measures of stability from AMMI model. Ann. Biol. Res. 3:3126-3136.

Zhang, Z., Lu C. and Xiang, Z.H. 1998. Analysis of variety stability based on AMMI model. Acta Agronomica Sinica 24:304-309.

\section{How to cite this article:}

Ajay Verma and Singh, G.P. 2020. GxE Interaction Analysis of Wheat Genotypes Evaluated under Restricted Irrigated Timely Sown Conditions of North Eastern Plains Zone using AMMI \& Yield Stability Measures. Int.J.Curr.Microbiol.App.Sci. 9(11): 957-970. doi: https://doi.org/10.20546/ijcmas.2020.911.114 